\title{
Descripción anatómica de la musculatura del miembro torácico del "carpincho" (Hydrochoerus hydrochaeris)
}

\author{
Cao, J.A.; Bode, F.F.; Fernández, J.A.; Resoagli, J.M. \\ Cátedra de Anatomía I, Facultad de Ciencias Veterinarias, UNNE. Sargento Cabral 2139, \\ Corrientes (3400), Argentina. Tel. 379-4425753, E-mail: jacao@vet.unne.edu.ar
}

\begin{abstract}
Resumen
Cao, J.A.; Bode, F.F.; Fernández, J.A.; Resoagli, J.M.: Descripción anatómica de la musculatura del miembro torácico del "carpincho" (Hydrochoerus hydrochaeris). Rev. vet. 26: 1, 38-42, 2015. El "carpincho" es un roedor de gran talla ampliamente distribuido en la zona de llanos y humedales de Sudamérica. La información anatómica sobre la musculatura de esta especie es escasa. El objetivo del ensayo fue describir las estructuras musculares de la extremidad torácica de Hydrochoerus hydrochaeris. Se utilizaron los miembros torácicos de cuatro cadáveres (hembras). Los especímenes fueron conservados mediante métodos anatómicos tradicionales. Tras separar los miembros, se retiró la piel, la grasa subcutánea y las fascias. Luego se realizó la disección de las diferentes regiones musculares, para determinar el origen e inserción de cada músculo, datos que se avalaron con registros fotográficos. Las observaciones revelaron que los músculos presentes son los mismos que los descriptos en otros mamíferos pentadáctiles, destacándose el gran desarrollo de los músculos de la región de la mano. Se espera que los estudios anatómicos de H. hydrochaeris contribuyan a mejorar su manejo sanitario y productivo.
\end{abstract}

Palabras clave: "carpincho", anatomía, músculos del miembro torácico.

\begin{abstract}
Cao, J.A.; Bode, F.F.; Fernández, J.A.; Resoagli, J.M.: Anatomical description of muscles from the thoracic limb of the "capybara" (Hydrochoerus hydrochaeris). Rev. vet. 26: 1, 38-42, 2015. "Capybara" is a great size rodent, widespread in plains and swamps of South America. The anatomical information on the musculature of this species is scarce. The objective of the assay was to describe the muscular structures of the thoracic limbs of the "capybara". The thoracic limbs of four female bodies were used. The specimens were preserved by traditional methods. The skin, subcutaneous fat and fascia of the thoracic limbs were removed. Dissection of the different muscle regions was performed to determine origins and insertions as well as digital photographic records. Observations revealed that these muscles are the same as those described in other pentadactil mammals, highlighting the great development of the muscles of the hand. The anatomical studies of H. Hydrochoerus will help to improve healthy production of the species.
\end{abstract}

Key words: "capybara", anatomy, muscles of thoracic limb.

\section{INTRODUCCIÓN}

El carpincho, también conocido como "capibara" o "chigüire" (Hydrochoerus hydrochaeris), posee en Sudamérica una amplia distribución que abarca desde Panamá hasta Argentina (sur de la Provincia de Buenos Aires). Diversas culturas precolombinas ya utilizaban esta especie de forma integral (carne, cuero) y -aún en nuestros días- constituyen una importante fuente proteica para poblaciones nativas y criollas ${ }^{2,9}$.
El usufructo sustentable de este recurso debe desarrollarse a la par de investigaciones científicas que lo respalden. En tal sentido, existen estudios detallados acerca de aspectos anatómicos del carpincho, así como datos fisiológicos sobre su reproducción y nutrición ${ }^{10}$.

Los músculos del miembro torácico de los mamíferos comprenden las regiones escapular, braquial, antibraquial y de la mano ${ }^{1,3-5}$. Dado que la información anatómica sobre las estructuras musculares del carpincho es escasa, el objetivo de este trabajo fue estudiar la miología del miembro torácico a fin de aportar conoci- 
mientos que contribuyan a mejorar las condiciones de crianza, manejo sanitario y conservación de la especie.

\section{MATERIAL Y MÉTODOS}

Se utilizaron los miembros torácicos de cuatro ejemplares adultos (hembras), con un peso muerto promedio de $45 \mathrm{~kg}$, decomisados por la Dirección de Flora y Fauna de la Provincia de Corrientes (Argentina) y donados a la Cátedra de Anatomía de la Facultad en la cual se realizaron los estudios. A los especimenes se les inyectó solución fijadora-conservadora vía arteria carótida común y se los dejó reposar envueltos en polietileno a temperatura de refrigeración.

Tras la extracción de los miembros torácicos, se retiró la piel, la grasa subcutánea y las fascias. Se realizó la disección de las regiones musculares para determinar el origen e inserción de los músculos. Los hallazgos fueron registrados fotográficamente con cámara fotográfica Kodak C 763 y la descripción se adaptó a los cánones de la nomenclatura establecida en la Nomina Anatómica Veterinaria ${ }^{8}$.

\section{RESULTADOS}

De las disecciones realizadas en la región escapular, se observó que el músculo deltoides (deltoideus) se caracterizó por tener dos porciones, acromiana y escapular, bien marcadas en su origen, las que se insertaron en la tuberosita deltoidea. El músculo supraespinoso (supraespinatus) ocupó la fosa homónima, se insertó por un tendón en el vértice del tubérculo mayor del húmero, donde presentó un hueso de naturaleza sesamoidea. El músculo infraespinoso (infraespinatus) se originó en la fosa infraespinosa, borde dorsal y caudal de la escápula, pasó bajo el proceso suprahamatus, al cual se fijó por medio de una lámina fibrosa y terminó por una rama profunda carnosa en la convexidad del tubérculo mayor y una rama superficial tendinosa en la fosa circular del tubérculo mayor del húmero. El músculo redondo menor (teres minor), cubierto por el musculo deltoides, nació en el tercio distal del borde (margo) caudal de la escápula y se insertó en una aponeurosis en la cresta del tubérculo mayor distal al músculo precedente (Figuras 1 y 2).

El músculo subescapular (subscapularis) ocupó la cara medial de la escápula. En su recorrido se fijó a la apófisis coracoides por una aponeurosis y su tendón terminal lo hizo en el tubérculo menor del húmero. El músculo coracobraquial (coracobrachialis) se caracterizó por tener un cuerpo carnoso, cuyo tendón se insertó en la cresta humeral, en distal de la tuberosidad deltoidea. El músculo redondo mayor (teres major), aplanado lateralmente, se originó en el ángulo y parte proximal del borde (margo) caudal de la escápula y por una aponeurosis se insertó en la depresión que reemplaza a la tuberosita teres (Figura 3).

En la región del brazo, el músculo bíceps braquial (biceps brachii) se originó por un tendón en la tube- rosidad supraglenoidea, se deslizó sobre la corredera bicipital (surco intertubercular) envuelto en una vaina sinovial dependiente de la cápsula articular y terminó en una aponeurosis en la ulna y por un delgado tendón en el tercio proximal del borde (margo) medial del radio. El músculo braquial (brachialis) tomó origen en proximal de la cara lateral del húmero, cruzó en dorsal de la superficie flexora de la articulación cubital y terminó por medio de una rama carnosa en dorsal del

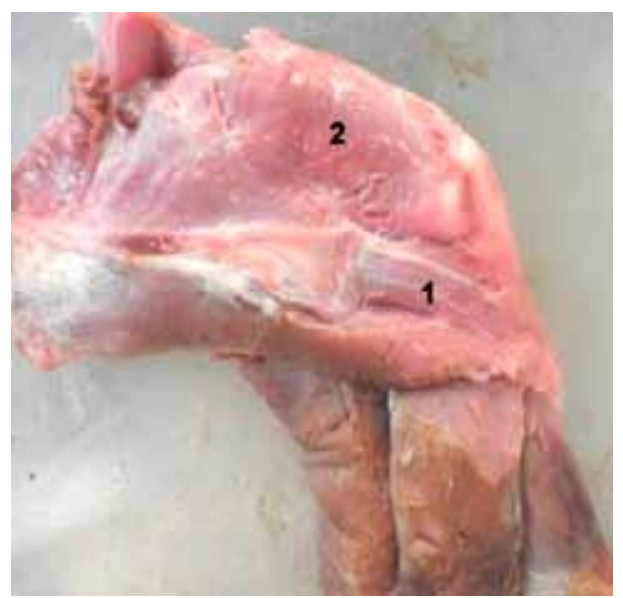

Figura 1. Músculos deltoides (1) y supraespinoso (2).

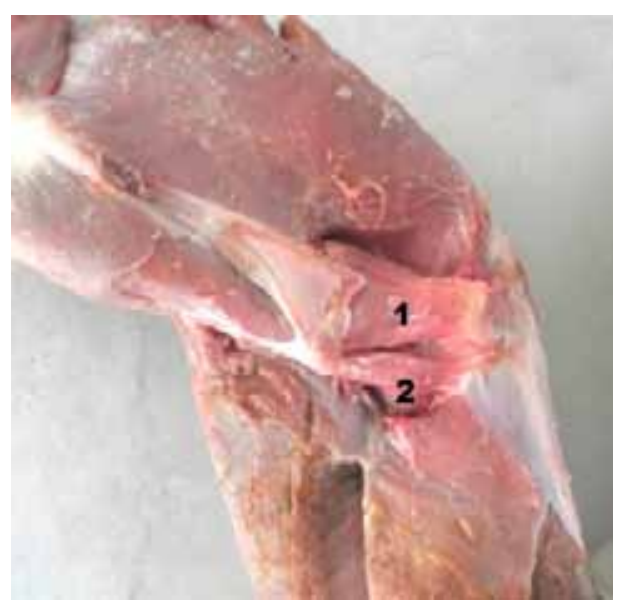

Figura 2. Músculos infraespinoso (1) y redondo menor (2).

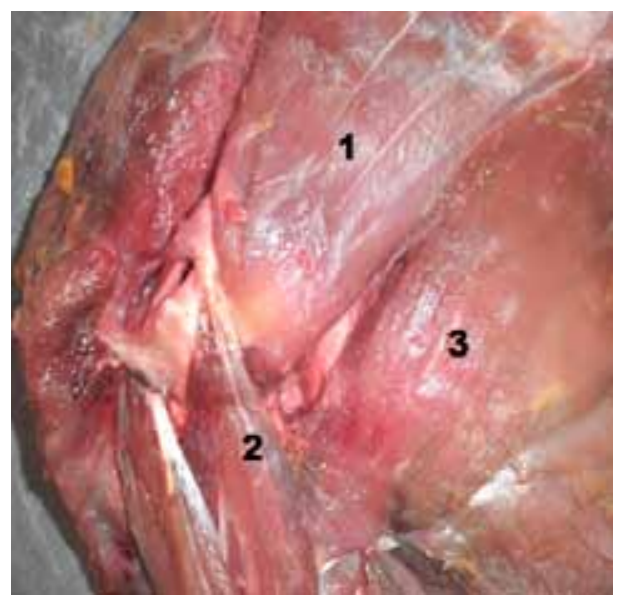

Figura 3. Músculos subescapular (1), coracobraquial (2) y redondo mayor (3). 
radio, en común con el músculo redondo pronador, enviando fibras entre los tendones del bíceps braquial, que se fijaron a la ulna (Figura 4).

El músculo tríceps braquial (tríceps brachii) se caracterizó por sus cuerpos carnosos voluminosos, bien diferenciados. La rama larga nació en el tercio distal del borde (margo) caudal de la escápula. La rama lateral se inició por una lámina fibrosa que tomó origen en

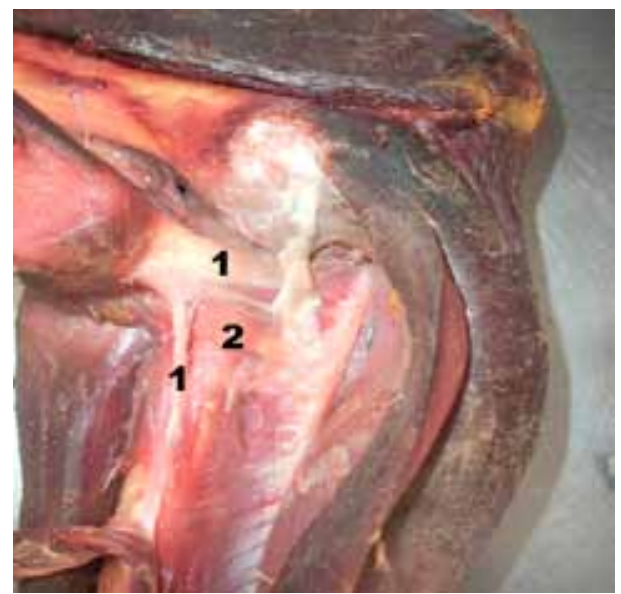

Figura 4. Músculos bíceps braquial (1) y braquial (2).

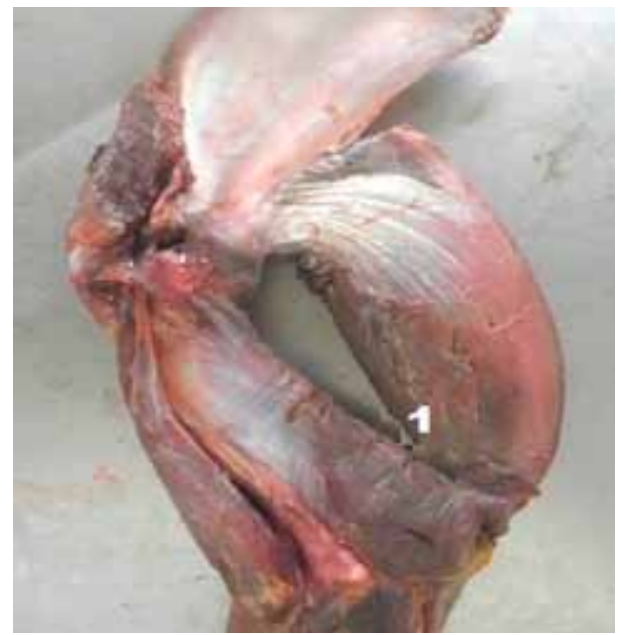

Figura 5. Músculo tríceps braquial (1), vista medial.

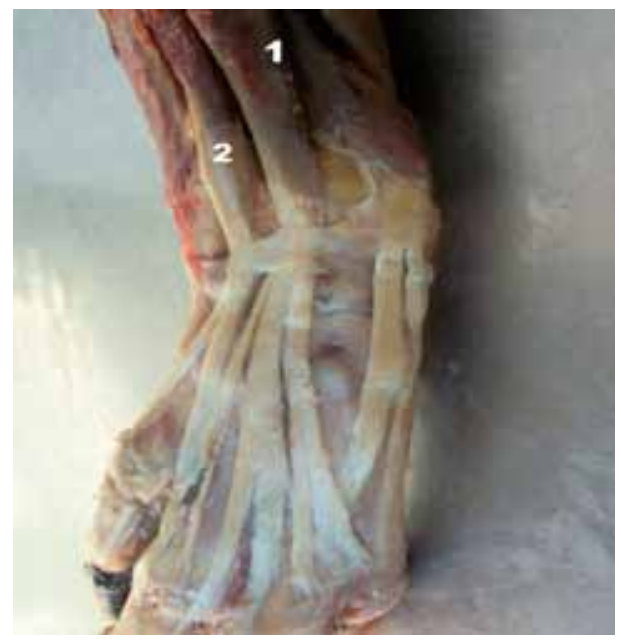

Figura 6. Músculos extensor digital común (1) y extensor digital lateral (2). la cresta humeral y la rama medial lo hizo en la parte proximal de la cara medial del húmero, cubierta por la aponeurosis del músculo teres mayor, las tres ramas se insertaron en el tubérculo olecraneano (Figura 5).

El músculo tensor de la fascia antibraquial (tensor fasciae antebrachii), largo y aplanado, tomó origen en la parte proximal de la cresta escapular, se adhirió en caudal y medial de la cabeza larga del tríceps braquial, y terminó a nivel del olécranon, donde se unió a la fascia antibraquial. En la región del antebrazo los músculos extensores del carpo y los dedos se ubicaron dorsolateralmente. Comenzando de dorsal en progresión palmar, el músculo extensor carpo radial (extensor carpi radialis) fue el primero, originado en la cresta supracondilar lateral del húmero. A nivel del tercio distal del radio se dividió en dos tendones que pasaron debajo del músculo abductor digital I largo y se fijaron a la cara dorsal de los huesos metacarpianos II y III.

El músculo extensor digital común (extensor digitalis communis), asentó en palmar del precedente, originándose en el epicóndilo lateral del húmero. A nivel del extremo distal del radio se dividió en tres tendones contenidos en una vaina sinovial, los cuales pasaron por la cara extensora del carpo e inmediatamente se separaron. El tendón medial terminó en tres ramas, una se fijó en dorsal de la falange distal del segundo dedo, las dos ramas restantes en la falange distal del tercer dedo. El tendón intermedio terminó en dos ramas en dorsal de la falange distal del tercer y cuarto dedo respectivamente. El tendón lateral se insertó en la falange distal del quinto dedo y emitió una rama que se unió al tendón medial del extensor digital lateral (Figuras 6 y 7$)$.

El músculo extensor digital lateral (extensor digitalis lateralis), parcialmente ubicado por debajo del músculo extensor digital común, se inició en el epicóndilo lateral del húmero. En distal del radio se dividió en dos tendones. El tendón lateral se fijó en la cara dorsal de la falange distal del quinto dedo, el medial en la falange proximal del cuarto dedo y recibió en su trayecto la rama lateral del extensor digital común (Figura 6).

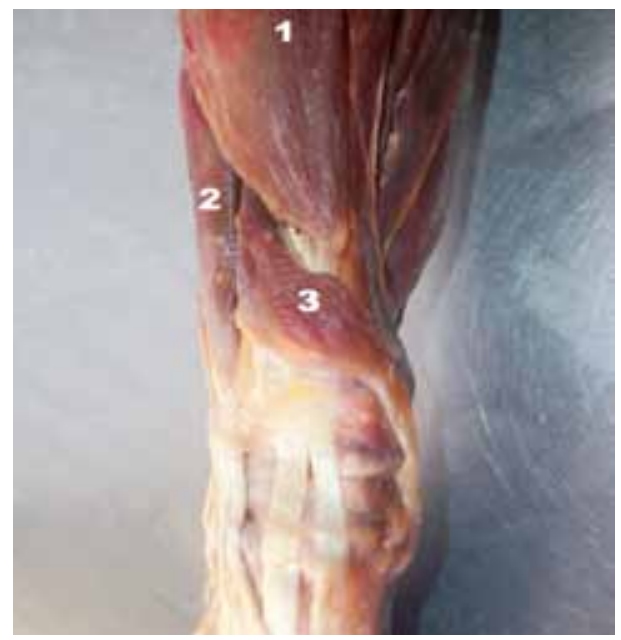

Figura 7. Músculos extensor carpo radial (1), extensor digital común (2) y abductor digital largo (3). 
El músculo extensor carpo ulnar (carpi ulnaris) nació en el epicóndilo lateral del húmero y cara lateral del olécranon, su tendón pasó por el surco lateral del extremo distal de la ulna y se insertó en el extremo proximal del metacarpo V. El músculo abductor digital I largo (abductor digiti I longus) se originó en lateral del radio y la ulna, cubierto por el extensor digital común; el cuerpo carnoso se deslizó superficialmente al músculo extensor carpo radial, formó un tendón que pasó por el surco medio del radio y se fijó al metacarpo I junto a una lámina fibrosa proveniente de la fascia antibraquial, la que se inició en el borde medial del radio, en distal de la inserción del músculo pronador teres (Figura 7).

El músculo supinador (supinator), unipenado, se originó por un pequeño tendón en el ligamento colateral y epicóndilo lateral del húmero y se insertó en la cara dorsal del radio, en proximal al músculo pronador teres. Los músculos antibraquiales palmares incluyeron a los flexores de la mano y de los dedos, junto a los pronadores. El músculo pronador teres (pronator teres) se inició por dos ramas, una en el epicóndilo medial y ligamento colateral y otra en proximal de la fosa coronoidea del húmero, insertándose por un tendón aplanado en la cara dorsal del radio (Figura 8).

El músculo flexor carpo radial (flexor carpi radialis), fusiforme, se originó en el epicóndilo medial del húmero, en caudal del pronador teres, y se insertó en el extremo proximal del metacarpo II. El músculo flexor carpo ulnar (flexor carpi ulnaris), con un cuerpo carnoso cónico, se originó en la cara medial del olécranon y por una aponeurosis en la parte caudolateral del mismo, terminando en un tendón en el hueso accesorio. El músculo flexor digital superficial (flexor digitalis superficialis) se originó en común con el músculo flexor digital profundo en el epicóndilo medial del húmero, dividiéndose en dos cuerpos carnosos, superficial y profundo. El superficial se continuó con una lámina fibrosa que cubrió la palma para terminar en íntima unión con la fascia de la mano. El cuerpo carnoso profundo se dividió en tres tendones, los cuales a nivel de los sesamoides proximales se separaron en dos ramas

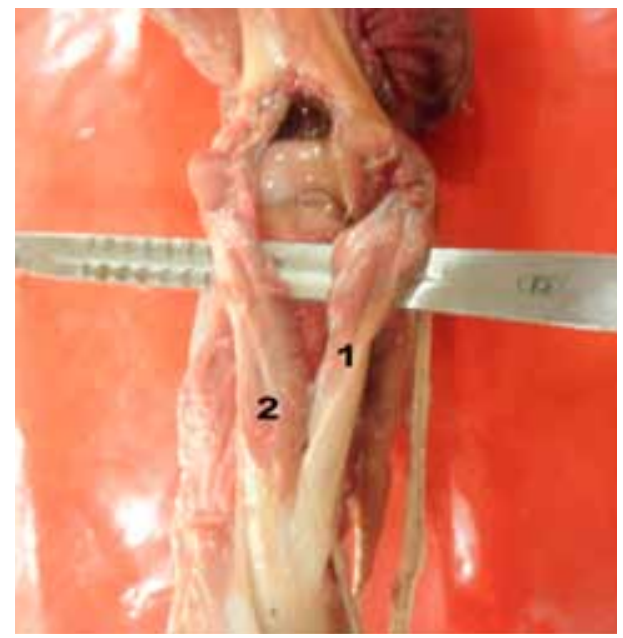

Figura 8. Músculos supinador (1) y pronador teres (2).

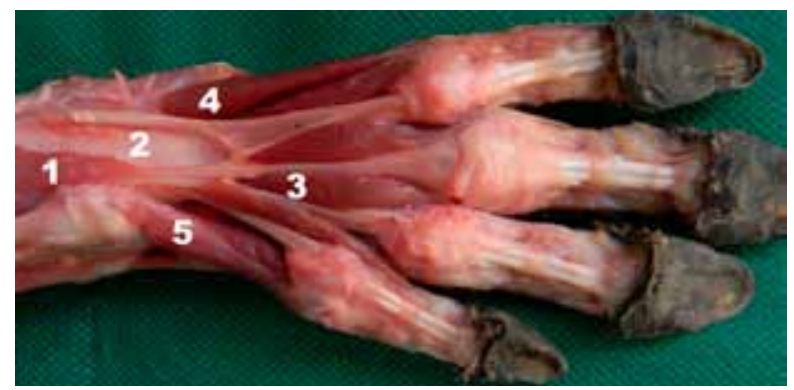

Figura 9. Músculos flexor digital superficial (1), flexor digital profundo (2), lumbricales (3) y abductores de los dedos II y V $(4,5)$.

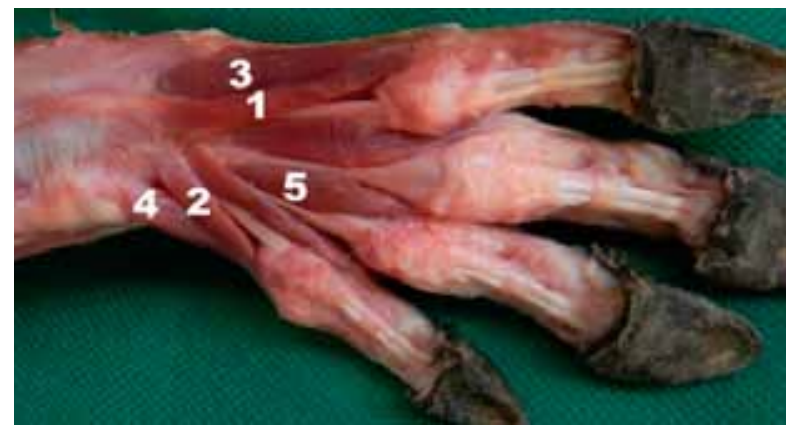

Figura 10. Músculos flexores digitales II y V $(1,2)$, abductores de los dedos II y V $(3,4)$ y lumbricales $(5)$.

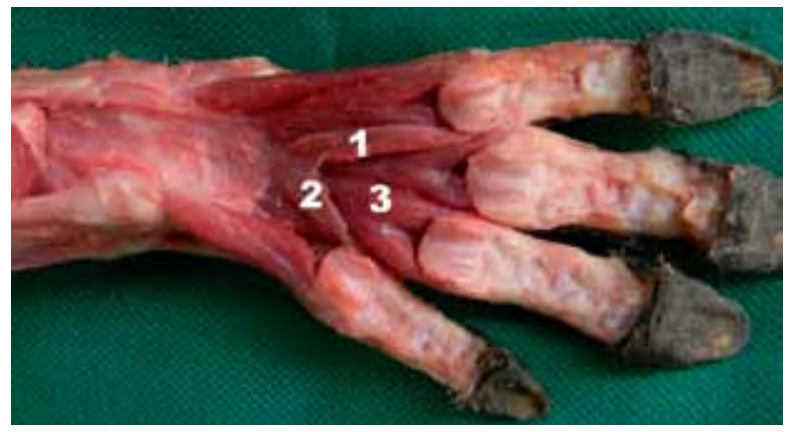

Figura 11. Músculos adductores de los dedos II y V (1, 2) e interóseos (3).

que se insertaron a ambos lados de las falanges intermedias de los dedos II, III y IV.

El músculo flexor digital profundo (flexor digitalis profundus) presentó dos cuerpos (venter) carnosos. El cuerpo humeral se conformó por dos porciones, una superficial voluminosa unida al flexor digital superficial y una profunda pequeña, en tanto que el cuerpo ulnar se originó en la cara medial del olécranon y borde caudal de la ulna, ramas que se unieron en un tendón común que deslizó por el canal del carpo y al salir se dividió en cuatro tendones, cada uno de los cuales -en la unión metacarpo falangiana- se dividió en dos ramas que se insertaron en la cara palmar de la falange distal de cada dedo (Figura 9).

El músculo pronador cuadrado (pronator quadratus) situado en toda la extensión de la arcada radio ulnar, se caracterizó por poseer fibras musculares con intersecciones fibrosas que se dirigieron distomedialmente desde la cara dorsal de la ulna al borde medial y surco de la cara palmar del radio. Dentro de los múscu- 
los de la mano se consideraron los propios de los dedos II y V. Los músculos flexores digitales II y V (flexor digiti II $y \mathrm{~V}$ ), se originaron en el ligamento carpo transverso y se insertaron en abaxial de la falange proximal del dedo correspondiente (Figura 10).

Los músculos adductores II y V (adductores digiti II $y \mathrm{~V}$ ) nacieron juntos en el ligamento carpo palmar profundo y se terminaron en axial de la base de la falange proximal de sus respectivos dedos. El músculo abductor digital II (abductor digiti II), cilíndrico, aguzado en su extremo distal, se originó en el ligamento colateral medial y borde del ligamento carpo palmar profundo y se insertó por un delgado tendón en abaxial de la base de la falange proximal del dedo II. El músculo abductor digital V (abductor digiti V), de forma piramidal, nació en el hueso accesorio y ligamento piso metacarpiano, terminando por un delgado tendón en abaxial de la base de la falange proximal. Los músculos lumbricales, tres pequeños músculos para los dedos III, $\mathrm{IV}$ y $\mathrm{V}$, se originaron en el punto de división del tendón del flexor digital superficial y se insertaron por una delgada lámina fibrosa en axial de la falange proximal de sus respectivos dedos (Figuras 9 y 10).

Los músculos interóseos, cuatro músculos (uno para cada dedo), se originaron en los huesos sesamoideos situados en la cara palmar de la articulación carpo metacarpiana, en la extremidad distal del metacarpo, dividiéndose en dos ramas musculares que se insertaron por un tendón en las caras abaxiales de los huesos sesamoideos de cada dedo, siguieron en dorsal y se unieron al tendón del músculo extensor digital común, en dorsal de la falange proximal (Figura 11).

\section{DISCUSIÓN}

Del estudio realizado surge que los músculos de las regiones escapular y braquial no revelaron diferencias dignas de mención, en relación con las descripciones realizadas en otros mamíferos domésticos ${ }^{1}$. En la región del antebrazo el desarrollo de los músculos pronadores y supinadores fue congruente con los de roedores y otras especies que presentan total independencia de los huesos del antebrazo.

Es dable destacar el desarrollo de los músculos de la región de la mano, la cual -al igual que en otras especies- estaba dotada con cuatro y cinco dedos, debido a la necesidad de trasladarse tanto en la tierra como en el agua ${ }^{11,12}$. En general, las observaciones efectuadas en la musculatura del miembro torácico del carpincho responden a las descripciones publicadas para los roedores en general ${ }^{2,6,7}$, con algunas particularidades que lo diferencian de otras especies.

\section{REFERENCIAS}

1. Barone R. 1976. Anatomia comparata dei mammiferi domestici, Vol. 1, Edagricole, Bologna, Italia.

2. Bolkovic ML, Ramadori D. 2006. Manejo de fauna silvestre en Argentina. Public. Secretaría de Ambiente y Desarrollo Sustentable, Buenos Aires, p. 168.

3. Bossi V, Caradonna GB, Spampani G, Varaldi L, Zimmerl U. 1909 Trattato de anatomia veterinaria, Vol. I, Ed. Vallardi, Milán, Italia, p. 610-642.

4. Getty R. 1982. Anatomía de los animales domésticos, $5^{\circ}$ ed., Interamericana, México, Tomo II, p. 1391-1394.

5. König-Liebich. 2001. Anatomía de los animales domésti$\cos , 2^{\mathrm{a}}$ ed., Tomo I: Aparato locomotor, Ed. Médica Panamericana, Madrid.

6. Mendoza TH. 1977. Tórax del chigüire (Hydrochoerus hydrochaeris hydrochaeris), cavidad, análisis y topografía de órganos y trayectos vasculo-nerviosos. Trabajo de Ascenso, Univ. Central Venezuela, 45 p.

7. Moreno V, Cardona J, Sánchez R, Acero D, Gordillo M. 2011. Descripción anatómica per se y particularidades osteológicas del esqueleto apendicular de Agouti paca. Rev CienciAgro (Journal de Ciencia y Tecnología Agraria, La Paz, Bolivia) 2: 279-290.

8. Nomina Anatomica Veterinaria. 2005. Published by the Editorial Committee, 5th. ed., Hannover, Germany.

9. Ojasti J. 1973. Estudio biológico del chigüire o capiba$r a$, Ed. Fondo Nacional de Investigaciones Agropecuarias, Caracas (Venezuela), p. 62-64.

10. Parker J, Haswell W. 1987. Zoología-Cordados, $7^{\circ}$ ed, Reverté, Barcelona, p. 107.

11. Pirlot P. 1976. Morfología evolutiva de los cordados, Omega, Barcelona, p. 291-293.

12. Romer A. 1973. Anatomía comparada, Interamericana, México, p.155-156. 